\title{
Comparing Common Average Referencing to Laplacian Referencing in Detecting Imagination and Intention of Movement for Brain Computer Interface
}

\author{
Syahrull Hi-Fi Syam ${ }^{1}$, Heba Lakany ${ }^{2}$, R.B. Ahmad ${ }^{3}$ and Bernard A. Conway ${ }^{2}$ \\ ${ }^{1}$ Polytechnic of Tuanku Syed Sirajuddin, 02600, Perlis, Malaysia \\ ${ }^{2}$ Biomedical Engineering, University of Strathclyde G4 0NW, Glasgow, United Kingdom. \\ ${ }^{3}$ Faculty of Informatics and Computing, Universiti Sultan Zainal Abidin (UniSZA), Kuala Terengganu, Malaysia
}

\begin{abstract}
Brain-computer interface (BCI) is a paradigm that offers an alternative communication channel between neural activity generated in the brain and the user's external environment. This paper investigates detection of intention of movement from surface EEG during actual and imagination of movement which is essential for developing non-invasive BCI system for neuro-impaired patients. EEG signal was recorded from 11 subjects while imagining and performing right wrist movement in multiple directions using 28 electrodes based on international 10-20 standard electrode placement locations. The recorded EEG signal later was filtered and pre-processed by spatial filter namely; Common average reference (CAR) and Laplacian (LAP) filter. Features were extracted from the filtered signal using ERSP and power spectrum and classified by $k$-nearest neighbour $(k$-NN) and quadratic discriminant analysis (QDA) classifiers. The classification results show that LAP filter has outperformed CAR with respect to classification. Classification accuracy ranged from $63.33 \%$ to $100 \%$ for detection of imagination of movement and $60 \%$ to $96.67 \%$ for detection of intention of actual movement. In both of detection of imagination and intention of movement k-NN classifier gave better result compared to QDA classifier.
\end{abstract}

\section{Introduction}

Neurological disorders such as amyotrophic lateral sclerosis (ALS), brainstem stroke, brain or spinal cord injury, cerebral palsy, muscular dystrophies and multiple sclerosis impair the neural pathway that control the muscle and /or the muscle themselves [1]. Patients loose voluntary control over their body due to these diseases and are driven to live an isolated, discriminated and restricted life in the long run. Their motor and sensory disabilities unable them to live a normal independent daily life.

Despite of losing their voluntary control over their body due to such neurological disorders, which is often a permanent effect, neuro-impaired patients can still communicate with outside world through brain-computer interface (BCI). BCI decodes the brain activities from electroencephalogram (EEG) signal and translates the user's intentions into commands [2] to control and/or communicate with the augmentative and assistive devices without activating any muscle or peripheral nerve.

In the past decades, number of BCI studies has been conducted in order to build successful interface that applies real-time EEG signal as command to control and /or communicate with the outside world [1]. Most of the studies aim to improve the speed and the accuracy of these interfaces by using improved EEG signal processing techniques and feature selection. One of the approaches that can be considered in signal processing techniques is implementing spatial and temporal filtering methods. The most often used spatial filtering methods in BCI studies to enhance the signal-to-noise ratio of EEG signal are Common average reference (CAR) [3, 4, 5] and Laplacian filter (LAP) [6, 7, 8].
In this paper we present the comparison of detecting motor imagery and intention of movement using 2 different spatial filters namely CAR and LAP. Our aim was to investigate which spatial filter would produce better results in detecting motor imagery and intention of movement.

The rest of the paper is structured as follow: Section 2 defines the implementation of experiment protocol and data analysis procedure. Section 3 presents the results of the experiment and the paper ends with a conclusion of the findings in section 4 .

\section{Method}

\subsection{Signal Enhancement by Spatial Filtering Methods}

EEG recordings are usually contaminated by several sources of artefacts produced by the subject (for example any minor body movement, electromyogram (EMG), electrocardiogram (ECG), eye movements, sweating). Also, technical glitches (for instance, power line, impedance fluctuation, cable movement, broken wire contact or excessive electrode paste/ dried pieces) add to the noise levels of the EEG signal [9]. Thus, it is important to remove any existing noise and artefact from the recorded EEG signal before implementing any further signal processing analysis. In this paper the EEG signal was filtered by spatial filter namely CAR and LAP.

CAR is one of the reference-free techniques that is not affected by problem associated with an actual physical reference [9]. In CAR, the potential at each electrode is measured with respect to the average of all 
electrodes. The CAR was computed using (1) given below [10]:

$$
V_{i}^{C A R}=V_{i}^{E R}-\frac{1}{n} \sum_{j=1}^{n} V_{j}^{E R}
$$

Where $V_{i}^{E R}$ is the potential between $i$ th electrode and the reference and $n$ is the number electrodes in the montage.

LAP serves as a high pass filter that enhances localized activity while suppressing the diffusion activity [8]. LAP is obtained by subtracting the sum of weighted potential of the surrounding electrodes from the current electrode potential where the weight is electrode distance dependent. The LAP was computed using (2) and (3) given below [10]:

$$
V_{i}^{L A P}=V_{i}^{E R}-\sum_{j \in S i}^{n} V_{j}^{E R} g_{i j}
$$

Where

$$
g_{i j}=\frac{1}{d_{i j}} / \sum_{j \in S i}^{n} \frac{1}{d_{i j}}
$$

$S i$ is the set of surrounding electrodes of the $i$ th electrode and $d i j$ is the distance between electrodes $i$ and $j$ (where $j$ is a member of $S i$ ).

\subsection{Experimental Recording Setup}

EEG signals were recorded from 11 subjects $(9$ males and 2 female) and the experimental procedure had been approved by the Departmental Ethics Committee of the Biomedical Engineering Department, University of Strathclyde.

Each subject faced a LCD monitor screen while being seated on a wheelchair at a distance of 1 meter from the screen. Subjects were attached to a manipulandum on to their right side. EEG and EMG recordings were acquired using Curry Neuroimaging Suite 7.0.8 XSB Software and NeuroScan ${ }^{\mathrm{TM}}$ Synamps $^{2}$ amplifiers from electrodes at 28 scalp locations and 4 bipolar electrodes (Figure 1). During the data recording process, subjects were required to hold the manipulandum and attempt, perform and imagine (kinesthetic imagery) performing right wrist movement (burst, point to point center out movement) towards multiple direction $(3,6,9,12)$ triggered by a visual cue.

The subject had to move the manipulandum rapidly in order to correspond to the cue direction shown in the monitor. On reaching the cue position, subjects had to hold the manipulandum at the cue position for as long as the cue remained visible on the screen and later reposition the manipulandum to the neutral position (0) according to the cue.

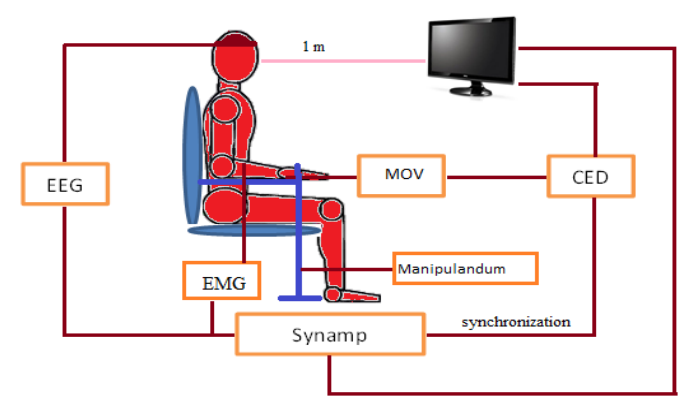

Figure 1: Experimental Recording Set Up.

The visual cue was displayed on the LCD monitor screen which consisted of 5 squares initially placed at the center of the screen whilst the subject held the manipulandum in the neutral position (0). The cue then randomly switched to different directions $(3,6,9,12)$ with a time interval of 10 seconds in between any 2 consecutive cues [11] (Figure 2).

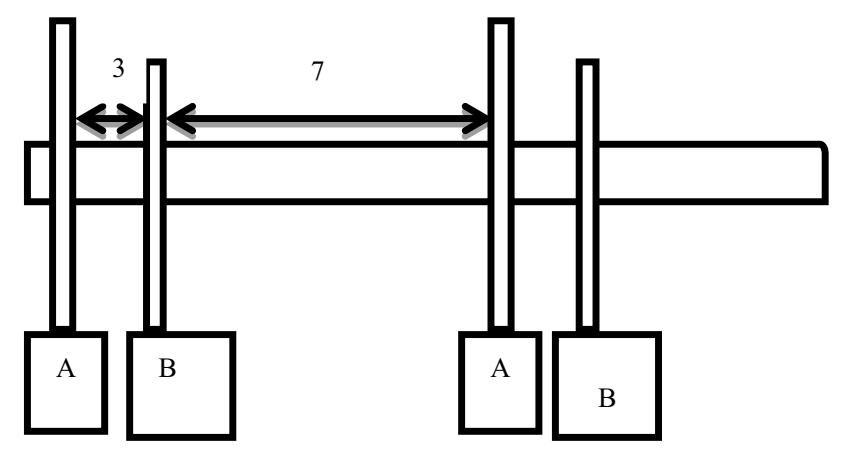

Figure 2: Timeline of Visual Cue Presentation.

In Fig. 2, during phase A, subjects were asked to move or imagine (kinesthetic imagery) moving the manipulandum from neutral position (0) to one of four directions $(3,6,9,12)$ and hold it in that position until the cue was visible on the screen; in phase B, subjects then repositioned the manipulandum back to the neutral position (0) as per the cue. While in the neutral position, subjects were instructed to stay calm and relaxed.

All the participants completed all the trials by imagining the movement and performing the actual movement based on external cue provided to them. Each experiment comprised of a total of 200 trials of both movement and imagery of movement towards 4 different directions, thus, establishing 50 repetitions per direction.

\subsection{Data Recording Set Up}

EEG, EMG and movement signals were recorded simultaneously during the experiments. EEG signal was recorded from 28 scalp locations located according to international 10-20 system (earlobe reference) using high density montage (Figure 3). EMG signals were recorded from flexor carpi radialis, extensor carpi ulnaris, extensor carpi radialis brevis and extensor carpi radialis longus muscles. The EMG signal was recorded in order to make sure that there is no movement during 
experiment of imagery of movement. Both EEG and EMG were recorded by Curry Neuroimaging Suite 7.0.8 XSB software using NeuroScan ${ }^{\mathrm{TM}}{ }_{\text {Synamps }}^{2}$ at sampling frequency of $2 \mathrm{KHz}$.

The movement signal was recorded from 2 precision servo potentiometers that are attached to the manipulandum in order to detect the onset of a movement. The signal recorded using Spike2 software through CED I401 (Cambridge Electronic Design, United Kingdom) at a sampling frequency of $100 \mathrm{~Hz}$.

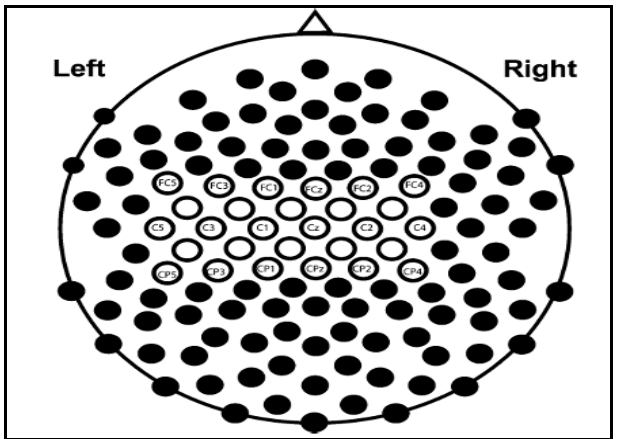

Figure 3: EEG Recording Montage.

\subsection{Data Pre-processing}

The recorded data from both of the experiments were processed offline. EEG was epoched using EEGLAB software version 12 [12] based on the type of experiments and categorized according to the type of the direction $(3,6,9,12)$. For instance, in experiment of external cue movement, the EEG signal was epoched 3 seconds before and 3 seconds after the onset of the movement (the movement initiation time) whereas, for the experiment where the subjects imagined the movement, the EEG signal was epoched 3 seconds before and 3 seconds after the initiation time (visual cue presentation time).

A Notch filter was applied to the epoched EEG to remove $50 \mathrm{~Hz}$ power line interference [13] and then a Chebyshev type 2 bandpass filter was used [14]. CAR and LAP methods were applied before the features were extracted.

\subsection{Features Extraction and Classification}

The features of the interest were Event Related Spectral Perturbation (ERSP) and average of Power Spectrum in alpha $(\alpha, 8-12 \mathrm{~Hz})$, beta $(\beta, 13-30 \mathrm{~Hz})$ and gamma $(\gamma, 31-$ $50 \mathrm{~Hz})$ frequency bands. ERSP is a generalization of Event Related Desynchronization (ERD)/Event Related Synchronization (ERS) which helps visualize the entire spectrum in form of baseline-normalized spectrogram. ERSP is computed where each epoch is divided into a number of overlapping windows and spectral power is calculated for each window. The calculated spectral power is then normalized (divided with the baseline spectra calculated from the EEG immediately before each event) and averaged over all the trials. The whole process was performed by EEGLAB [12]. Power Spectrum indicates the distribution level of the signal power in frequency. Average of Power Spectrum in $\alpha, \beta$ and $\gamma$ frequency bands were computed using code adapted from the EEGLAB.

Features were extracted from neutral condition and motor imagery/ intention of movement condition for direction of $3,6,9$ and 12 respectively. For neutral condition, features were extracted $500 \mathrm{~ms}$ before the subject imagined/performed the wrist movement. On the other hand, for motor imagery, features were extracted $500 \mathrm{~ms}$ after the initiation time $(\mathrm{t}=0)$ whereas for intention of movement features were extracted $500 \mathrm{~ms}$ prior to the onset of the movement $(t=0)[15,16]$. The extracted features of the epoched EEG were spilt into training data set $(70 \%)$ and testing data set $(30 \%)$ $[19,21]$. The training data set and testing data set are randomly $[19,23]$ selected by using Matlab function 'crossvalind' and fed to $k$-Nearest Neighbors $(k-\mathrm{NN})$ $[17,18]$ and quadratic discriminant analysis (QDA) $[19$, $20]$ classifier for pattern recognition classification. For $k$ NN classifier, ' $k$ ' was set to 8 [22].

\section{Results}

\subsection{Results of Event Related Spectral Perturbation (ERSP)}

Figure 4,5,6 and 7 show the ERSP results for detection of motor imagery and intention of movement for direction 3, 6, 9 and 12 at channel C3 using CAR and LAP filters respectively. The results are obtained from subject 1 for both of experiments.

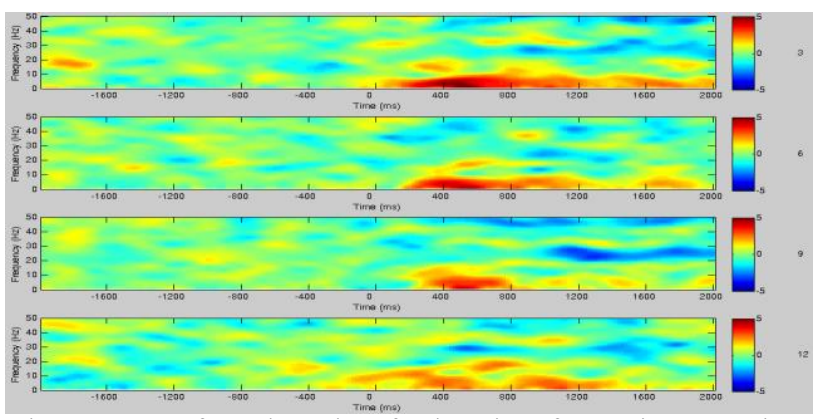

Figure 4: ERSP from channel C3 for detection of motor imagery using CAR Method.

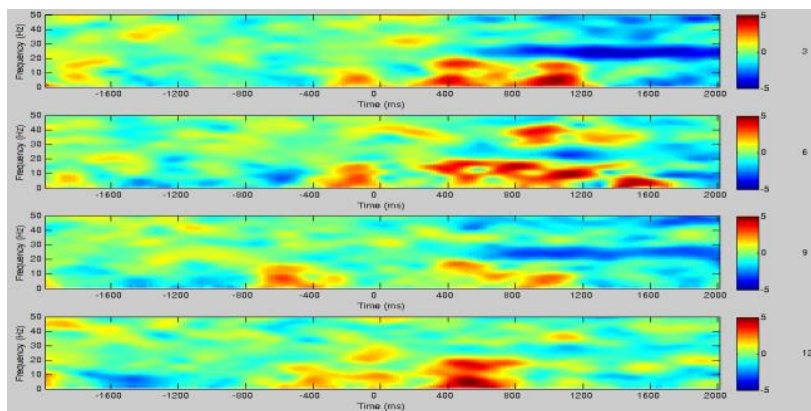

Figure 5: ERSP from channel C3 for detection of motor imagery using LAP Method

From Figures 4 and 5, ERSP using LAP method show more significant result when compared to CAR method in detection of motor imagery towards direction $3,6,9$ and 12. The decrease in power spectrum as shown by the ERD (blue colour) $400 \mathrm{~ms}$ after the initiation time $(\mathrm{t}=0)$ for all directions. ERD was evidently detected in $\beta$ 
(direction 3, 6, 9 and 12) and $\gamma$ band (direction 3, 6 and 9).

Also from Figure 6 and 7, ERSP using LAP method again shows more significant result compared to CAR method in detection of intention of movement towards direction 3, 6, 9 and 12. In this paper, intention of movement refers to $500 \mathrm{~ms}$ prior to the onset of the movement $(\mathrm{t}=0)$. ERD was clearly detected in $\alpha(6,9$ and 12) and $\gamma$ band (12, 9 and 3$)$.

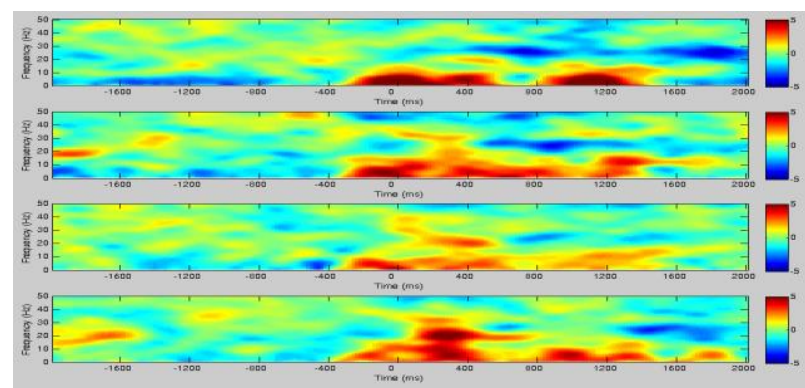

Figure 6: ERSP of channel C3 for detection of intention of movement using CAR Method.

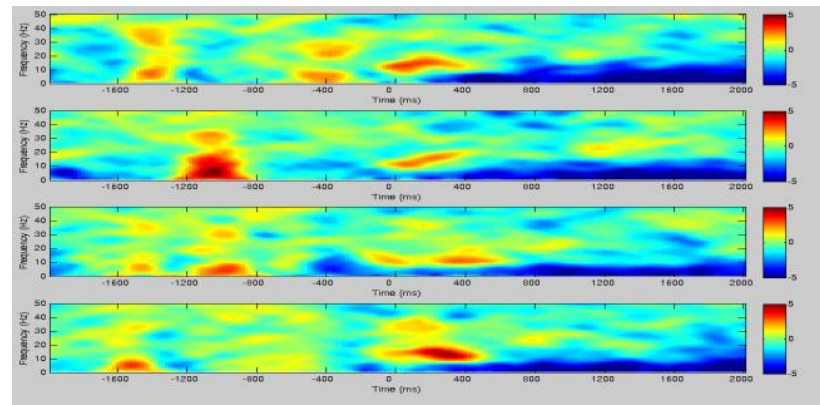

Figure 7: ERSP of channel C3 for detection of intention of movement using LAP Method.

\subsection{Results of Power Spectrum}

The results for detection of motor imagery are presented in Table 1 and Table 2, while results for detection intention of movement are tabulated in Table 3 and Table 4. The extracted features using CAR method and LAP method for direction toward 3, 6, 9 and 12 in detection of motor imagery and intention of movement are classified using $k$-NN and QDA classifier. In each of the table presented the maximum classification accuracy (Acc), channel of the maximum classification accuracy $(\mathrm{Ch})$, true positive rate (TPR) and false positive rate (FPR).

TABLE 1: Classification results of detection of motor imagery for direction toward 3, 6, 9 and 12 using $k$-NN classifier on average of Power Spectrum in alpha $(\alpha)$, beta $(\beta)$ and gamma $(\gamma)$ band feature.

\begin{tabular}{|c|c|c|c|c|c|c|c|c|c|}
\hline \multirow{3}{*}{ 总 } & \multirow{3}{*}{ 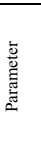 } & \multicolumn{8}{|c|}{$\begin{array}{l}\text { Maximum Classification Accuracy, True Positive Rate (TPR) and False Positive Rate (FPR) } \\
\text { for direction 3, 6, } 9 \text { and } 12(\%)\end{array}$} \\
\hline & & \multicolumn{2}{|c|}{ Direction 3} & \multicolumn{2}{|c|}{ Direction 6} & \multicolumn{2}{|c|}{$\begin{array}{l}\text { Direction } 9 \\
\end{array}$} & \multicolumn{2}{|c|}{ Direction 12} \\
\hline & & CAR & LAP & CAR & LAP & CAR & LAP & CAR & LAP \\
\hline \multirow{4}{*}{$\bar{n}$} & Acc & 73.33 & 83.33 & 76.67 & 80.00 & 76.67 & 86.67 & 76.67 & 80.00 \\
\hline & $\mathrm{Ch}$ & $\mathrm{CCP} 3$ & FC5 & FCZ & $\mathrm{C} 3$ & CCP4 & CFC5 & CP2 & CFCl \\
\hline & TPR & 66.67 & 80.00 & 93.33 & 93.33 & 80.00 & 80.00 & 93.33 & 66.67 \\
\hline & FPR & 20.00 & 13.33 & 40.00 & 33.33 & 26.67 & 6.67 & 40.00 & 6.67 \\
\hline \multirow{4}{*}{$\approx$} & Acc & 83.33 & 90.00 & 86.67 & 93.33 & 83.33 & 93.33 & 83.33 & 96.67 \\
\hline & $\mathrm{Ch}$ & $\mathrm{CFC1}$ & CFC1 & CFC1 & CFC1 & CFCl & $\begin{array}{l}\mathrm{CCP} 4 \\
\end{array}$ & $\mathrm{CFC1}$ & CFC1 \\
\hline & TPR & 73.33 & 86.67 & 80.00 & 93.33 & 66.67 & 86.67 & 73.33 & 93.33 \\
\hline & FPR & 6.67 & 6.67 & 6.67 & 6.67 & 0 & 0 & 6.67 & 0 \\
\hline
\end{tabular}

\begin{tabular}{|c|c|c|c|c|c|c|c|c|c|}
\hline \multirow{4}{*}{$\tilde{n}$} & Acc & 83.33 & 86.67 & 73.33 & 80.00 & 83.33 & 76.67 & 73.33 & 76.67 \\
\hline & $\mathrm{Ch}$ & $\mathrm{C} 4$ & FC5 & CP4 & CFC2 & $\mathrm{C} 4$ & CFC4 & $\mathrm{FC1}$ & CCP3 \\
\hline & TPR & 86.67 & 93.33 & 53.33 & 80.00 & 86.67 & 73.33 & 73.33 & 73.33 \\
\hline & FPR & 20.00 & 20.00 & 6.67 & 20.00 & 20.00 & 20.00 & 26.67 & 20.00 \\
\hline & Acc & 73.33 & 80.00 & 76.67 & 80.00 & 80.00 & 83.33 & 76.67 & 93.33 \\
\hline & $\mathrm{Ch}$ & $\mathrm{CZ}$ & C3 & CP4 & C3 & $\mathrm{CZ}$ & FC5 & $\mathrm{FC4}$ & $\mathrm{FC4}$ \\
\hline$\omega$ & TPR & 80.00 & 73.33 & 66.67 & 66.67 & 60.00 & 80.00 & 66.67 & 93.33 \\
\hline & FPR & 33.33 & 13.33 & 13.33 & 6.67 & 0 & 13.33 & 13.33 & 6.67 \\
\hline \multirow{4}{*}{$\approx$} & Acc & 73.33 & 96.67 & 73.33 & 80.00 & 76.67 & 80.00 & 83.33 & 86.67 \\
\hline & $\mathrm{Ch}$ & FC4 & $\begin{array}{l}\text { CFC4 } \\
\text {. }\end{array}$ & $\overline{\mathrm{FC} 3}$ & $\mathrm{CCP} 1$ & CP1 & CCP1 & FC3 & CFC2 \\
\hline & TPR & 60.00 & 93.33 & 66.67 & 73.33 & 66.67 & 100 & 86.67 & 80.00 \\
\hline & FPR & 13.33 & 0 & 20.00 & 13.33 & 13.33 & 40.00 & 20.00 & 6.67 \\
\hline \multirow{4}{*}{ ๓ } & Acc & 70.00 & 80.00 & 73.33 & 86.67 & 73.33 & 80.00 & 73.33 & 90.00 \\
\hline & $\mathrm{Ch}$ & $\mathrm{CP} 4$ & $\mathrm{C} 4$ & FCZ & $\mathrm{CCP} 3$ & $\overline{\mathrm{CCP} 2}$ & CCP3 & $\mathrm{C} 3$ & $\begin{array}{l}\mathrm{CP} 3 \\
\end{array}$ \\
\hline & TPR & 66.67 & 86.67 & 66.67 & 80.00 & 66.67 & 80.00 & 80.00 & 80.00 \\
\hline & FPR & 26.67 & 26.67 & 20.00 & 6.67 & 20.00 & 20.00 & 33.33 & 6.67 \\
\hline \multirow{4}{*}{ is } & Acc & 70.00 & 83.33 & 70.00 & 93.33 & 73.33 & 96.67 & 73.33 & 93.33 \\
\hline & $\mathrm{Ch}$ & CP3 & CFC3 & CP3 & $\mathrm{FC} 2$ & CCP4 & CP3 & ССР3 & $\mathrm{FC} 3$ \\
\hline & TPR & 80.00 & 93.33 & 80.00 & 100 & 66.67 & 93.33 & 66.67 & 93.33 \\
\hline & FPR & 40.00 & 26.67 & 40.00 & 13.33 & 20.00 & 0 & 20.00 & 6.67 \\
\hline \multirow{4}{*}{$\stackrel{\infty}{\infty}$} & Acc & 73.33 & 93.33 & 70.00 & 100 & 73.33 & 90.00 & 70.00 & 90.00 \\
\hline & Ch & CFC5 & CP2 & C3 & $\mathrm{FC} 1$ & $\mathrm{C} 4$ & CP3 & $\mathrm{CPC} 3$ & FC5 \\
\hline & TPR & 73.33 & 93.33 & 73.33 & 100 & 86.67 & 100 & 80.00 & 80.00 \\
\hline & FPR & 26.67 & 6.63 & 33.33 & 0 & 40.00 & 20.00 & 40.00 & 0 \\
\hline \multirow{4}{*}{ के } & $\overline{A c c}$ & 73.33 & 76.67 & 73.33 & 76.67 & 73.33 & 83.33 & 73.33 & 76.67 \\
\hline & $\mathrm{Ch}$ & CP5 & CFC5 & $\mathrm{FCl}$ & CP5 & $\mathrm{FC1}$ & CCP5 & $\mathrm{CFC} 3$ & CFC5 \\
\hline & TPR & 60.00 & 80.00 & 53.33 & 80.00 & 86.67 & 86.67 & 53.33 & 80.00 \\
\hline & FPR & 13.33 & 26.67 & 7.14 & 26.67 & 31.58 & 20.00 & 6.67 & 26.67 \\
\hline \multirow{4}{*}{$\frac{O}{n}$} & Acc & 70.00 & 90.00 & 83.33 & 86.67 & 70.00 & 86.67 & 73.33 & 90.00 \\
\hline & $\mathrm{Ch}$ & CP1 & C3 & $\mathrm{C} 4$ & CCP5 & CCP5 & $\mathrm{FC} 3$ & CCP5 & C3 \\
\hline & TPR & 73.33 & 86.67 & 100 & 73.33 & 60.00 & 100 & 73.33 & 86.67 \\
\hline & FPR & 33.33 & 6.67 & 33.33 & 0 & 20.00 & 26.67 & 26.67 & 6.67 \\
\hline \multirow{4}{*}{$\overline{\bar{n}}$} & Acc & 70.00 & 90.00 & 76.67 & 83.33 & 70.00 & 93.33 & 76.67 & 83.33 \\
\hline & $\mathrm{Ch}$ & $\mathrm{C} 2$ & CP5 & $\mathrm{CPZ}$ & FC5 & $\mathrm{C} 2$ & $\mathrm{CP} 4$ & $\mathrm{CFC} 3$ & $\mathrm{CZ}$ \\
\hline & TPR & 80.00 & 93.33 & 66.67 & 86.67 & 66.67 & 86.67 & 100 & 86.67 \\
\hline & $\overline{\text { FPR }}$ & 40.00 & 13.33 & 13.33 & 20.00 & 26.67 & 0 & 46.67 & 20.00 \\
\hline
\end{tabular}

Tabulated data in Table 1 shows the classification results of direction toward 3, 6, 9 and 12 for detection of motor imagery, dwell within the range of $70.00 \%$ to $100.00 \%$. Maximum classification accuracy for these four directions are obtained from different electrodes, namely CFC4 with classification accuracy of $96.67 \%$ (direction toward 3), FC1 with classification accuracy of $100.00 \%$ (direction toward 6), CP3 with classification accuracy of $96.67 \%$ (direction toward 9) and CFC1 with classification accuracy of $96.67 \%$ (direction toward 12). In all four directions, features extracted using spatial filter LAP contributed for the maximum classification results.

TABLE 2: Classification results for detection of motor imagery for direction toward 3, 6, 9 and 12 using QDA classifier on average of Power Spectrum in alpha $(\alpha)$, beta $(\beta)$ and gamma $(\gamma)$ band feature.

\begin{tabular}{|c|c|c|c|c|c|c|c|c|c|}
\hline \multirow{3}{*}{$\stackrel{\overline{\mathscr{e}}}{\stackrel{\Xi}{\Xi}}$} & \multirow{3}{*}{ 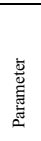 } & \multicolumn{8}{|c|}{$\begin{array}{l}\text { Maximum Classification Accuracy, True Positive Rate (TPR) and False Positive Rate (FPR) for } \\
\text { direction 3, } 6,9 \text { and } 12(\%)\end{array}$} \\
\hline & & \multicolumn{2}{|c|}{ Direction 3} & \multicolumn{2}{|c|}{ Direction 6} & \multicolumn{2}{|c|}{ Direction 9} & \multicolumn{2}{|c|}{ Direction 12} \\
\hline & & CAR & LAP & CAR & LAP & CAR & LAP & CAR & LAP \\
\hline \multirow[b]{4}{*}{$\bar{n}$} & Acc & 73.33 & 73.33 & 66.67 & 70.00 & 66.67 & 83.33 & 73.33 & 80.00 \\
\hline & $\mathrm{Ch}$ & $\mathrm{CP} 1$ & $\mathrm{C} 3$ & $\mathrm{FC} 1$ & $\overline{\mathrm{CCP} 1}$ & $\mathrm{C} 4$ & CFC5 & CFC4 & CFC1 \\
\hline & TPR & 93.33 & 86.67 & 86.67 & 53.33 & 86.67 & 80.00 & 53.33 & 80.00 \\
\hline & FPR & 46.67 & 40.00 & 53.33 & 13.33 & 53.33 & 13.33 & 6.67 & 20.00 \\
\hline \multirow{4}{*}{ బ } & Acc & 83.33 & 93.33 & 86.67 & 96.67 & 83.33 & 93.33 & 83.33 & 93.33 \\
\hline & $\mathrm{Ch}$ & CFC1 & CFC1 & CFC1 & CFC1 & FCZ & CFC1 & $\mathrm{FC} 2$ & CFC1 \\
\hline & TPR & 73.33 & 100 & 86.67 & 100 & 73.33 & 100 & 80.00 & 86.67 \\
\hline & FPR & 6.67 & 13.33 & 13.33 & 6.67 & 6.67 & 13.33 & 13.33 & 0 \\
\hline \multirow[b]{4}{*}{$\tilde{n}$} & Acc & 76.67 & 90.00 & 76.67 & 73.33 & 73.33 & 76.67 & 73.33 & 73.33 \\
\hline & $\mathrm{Ch}$ & CFC5 & CFC5 & CP4 & CFC4 & $\mathrm{FC} 2$ & CFC4 & CFC2 & CFC4 \\
\hline & TPR & 86.67 & 80.00 & 73.33 & 53.33 & 80.00 & 60.00 & 66.67 & 60.00 \\
\hline & FPR & 33.33 & 0 & 20.00 & 6.67 & 33.33 & 6.67 & 20.00 & 13.33 \\
\hline
\end{tabular}




\begin{tabular}{|c|c|c|c|c|c|c|c|c|c|}
\hline \multirow{4}{*}{ 蕅 } & Acc & 70 & 76.67 & 73.33 & 73.33 & 76.67 & $\overline{80.00}$ & 80.00 & 86.67 \\
\hline & $\mathrm{Ch}$ & CP5 & $\begin{array}{l}\text { CPP5 } \\
\end{array}$ & $\mathrm{CP} 4$ & $\mathrm{C} 3$ & $\mathrm{CP} 2$ & $\mathrm{FC1}$ & $\mathrm{FC} 4$ & $\mathrm{C} 2$ \\
\hline & TPR & 53.33 & 80.00 & 60.00 & 53.33 & 66.67 & 80.00 & 80.00 & 80.00 \\
\hline & FPR & 13.33 & 26.67 & 13.33 & 6.67 & 13.33 & 20.00 & 20.00 & 6.67 \\
\hline \multirow[b]{4}{*}{$n$} & Acc & 76.67 & 90.00 & 73.33 & 73.33 & 70.00 & 86.67 & 76.67 & 80.00 \\
\hline & $\mathrm{Ch}$ & $\mathrm{C} 4$ & CFC3 & $\mathrm{CFC1}$ & CCP1 & $\mathrm{FC4}$ & $\mathrm{CCP} 1$ & $\mathrm{C} 3$ & FC3 \\
\hline & TPR & 60.00 & 86.67 & 53.33 & 66.67 & 66.67 & 93.33 & 66.67 & 80.00 \\
\hline & $\begin{array}{l}\text { FPR } \\
\end{array}$ & 6.67 & 6.67 & 6.67 & 20.00 & 20.00 & 20.00 & 13.33 & 20.00 \\
\hline \multirow[b]{4}{*}{ œ } & Acc & 63.33 & 80.00 & 70.00 & 80.00 & 70.00 & 76.67 & 73.33 & 83.33 \\
\hline & $\mathrm{Ch}$ & FC1 & $\overline{\mathrm{C} 2}$ & $\mathrm{CPZ}$ & FC5 & CFC4 & $\overline{\mathrm{CFCl}}$ & $\mathrm{CCP} 1$ & CFC1 \\
\hline & TPR & 66.67 & 73.33 & 53.33 & 80.00 & 60.00 & 66.67 & 86.67 & 86.67 \\
\hline & FPR & 40.00 & 13.33 & 13.33 & 20.00 & 20.00 & 13.33 & 40.00 & 20.00 \\
\hline \multirow[b]{4}{*}{ in } & Acc & 63.33 & 80.00 & 70.00 & 80.00 & 70.00 & 76.67 & 73.33 & 83.33 \\
\hline & $\mathrm{Ch}$ & FC1 & $\mathrm{C} 2$ & $\mathrm{CPZ}$ & FC5 & $\mathrm{CFC4}$ & $\mathrm{CFCl}$ & $\mathrm{CCP} 1$ & CFC1 \\
\hline & TPR & 66.67 & 73.33 & 53.33 & 80.00 & 60.00 & 66.67 & 86.67 & 86.67 \\
\hline & FPR & 40.00 & 13.33 & 13.33 & 20.00 & 20.00 & 13.33 & 40.00 & 20.00 \\
\hline \multirow[b]{4}{*}{$\stackrel{\infty}{\infty}$} & Acc & 70.00 & 90.00 & 70.00 & 96.67 & $\begin{array}{l}76.67 \\
\end{array}$ & 90.00 & 70.00 & 83.33 \\
\hline & $\mathrm{Ch}$ & $\mathrm{C} 1$ & $\mathrm{FC} 1$ & CCP1 & CP3 & C4 & CP2 & $\mathrm{C} 2$ & CP3 \\
\hline & TPR & 86.67 & 86.67 & 80.00 & 93.33 & 86.67 & 86.67 & 86.67 & 73.33 \\
\hline & FPR & 46.67 & 6.67 & 40.00 & 0 & 33.33 & 6.67 & 46.67 & 6.67 \\
\hline \multirow[b]{4}{*}{$\ddot{m}$} & Acc & 70.00 & 76.67 & 70.00 & 76.67 & 70.00 & 80.00 & 73.33 & 73.33 \\
\hline & $\mathrm{Ch}$ & $\mathrm{CFC} 3$ & $\mathrm{FC} 1$ & CP3 & CP5 & $\mathrm{CFC} 3$ & $\mathrm{CFCl}$ & $\mathrm{FC} 3$ & CCP1 \\
\hline & TPR & 80.00 & 73.33 & 73.33 & 80.00 & 53.33 & 66.67 & 60.00 & 80.00 \\
\hline & FPR & 40.00 & 20.00 & 33.33 & 26.67 & 13.33 & 6.67 & 13.33 & 33.33 \\
\hline \multirow{4}{*}{$\frac{\circ}{\omega}$} & Acc & 70.00 & 93.33 & 86.67 & 93.33 & 70.00 & 90.00 & 70.00 & 96.67 \\
\hline & $\mathrm{Ch}$ & CCP5 & $\mathrm{C} 2$ & CP3 & CCP5 & CFC5 & $\mathrm{C} 2$ & CCP5 & CCP5 \\
\hline & TPR & 66.67 & 93.33 & 86.67 & 93.33 & 93.33 & 86.67 & 66.67 & 100 \\
\hline & FPR & 26.67 & 6.67 & 13.33 & 6.67 & 13.33 & 6.67 & 26.67 & 6.67 \\
\hline \multirow{4}{*}{$\overline{\bar{w}}$} & Acc & 73.33 & 96.67 & 70.00 & 83.33 & 70.00 & 86.67 & 70.00 & 83.33 \\
\hline & $\overline{\mathrm{Ch}}$ & FC5 & $\mathrm{CPZ}$ & $\overline{C P Z}$ & CFC3 & $\mathrm{CZ}$ & CP2 & CFC1 & CFC3 \\
\hline & TPR & 100 & 100 & 60.00 & 80.00 & 73.33 & 80.00 & 80.00 & 93.33 \\
\hline & FPR & 33.33 & 6.67 & 20.00 & 13.33 & 33.33 & 6.67 & 40.00 & 26.67 \\
\hline
\end{tabular}

Referring to Table 2, the classification results toward four directions for detection of motor imagery lie within the range of $63.33 \%$ to $96.67 \%$. Maximum classification accuracies for direction toward 3, 9 and 12 were obtained from electrode CFC1 with classification accuracy of $93.33 \%$. On the other hand, maximum classification accuracy for direction toward 6 was obtained from electrodes $\mathrm{CP} 3$ and $\mathrm{CFC} 1$ with classification accuracy of $96.67 \%$. The features extracted using spatial filter LAP contributed for the maximum classification results in all direction.

TABLE 3: Classification results of detection intention of movement for direction toward 3, 6, 9 and 12 using $k$ NN classifier on average of Power Spectrum in alpha $(\alpha)$, beta $(\beta)$ and gamma $(\gamma)$ band feature.

\begin{tabular}{|c|c|c|c|c|c|c|c|c|c|}
\hline \multirow{3}{*}{$\begin{array}{l}\frac{\bar{o}}{0} \\
\overline{0} \\
\bar{n}\end{array}$} & \multirow{3}{*}{ 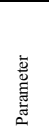 } & \multicolumn{8}{|c|}{ for direction 3, 6, 9 and $12(\%)$} \\
\hline & & \multicolumn{2}{|c|}{ Direction 3} & \multicolumn{2}{|c|}{ Direction 6} & \multicolumn{2}{|c|}{ Direction 9} & \multicolumn{2}{|c|}{ Direction 12} \\
\hline & & CAR & LAP & $\overline{\mathrm{CAR}}$ & LAP & CAR & LAP & CAR & LAP \\
\hline \multirow[b]{4}{*}{$\bar{\omega}$} & Acc & 76.67 & 83.33 & 76.67 & 80.00 & 80.00 & 80.00 & 76.67 & 80.00 \\
\hline & $\mathrm{Ch}$ & $\mathrm{CCP} 4$ & $\mathrm{FCl}$ & $\mathrm{CCP} 4$ & FC5 & FC4 & FC2 & $\mathrm{C} 3$ & $\mathrm{C} 1$ \\
\hline & TPR & 80.00 & 66.67 & 86.67 & 73.33 & 86.67 & 80.00 & 60.00 & 73.33 \\
\hline & FPR & 26.67 & 0 & 23.33 & 13.33 & 26.67 & 20.00 & 13.33 & 13.33 \\
\hline \multirow[b]{4}{*}{$\tilde{\omega}$} & Acc & 90.00 & 93.33 & 96.67 & 90.00 & 93.33 & 93.33 & 90.00 & 93.33 \\
\hline & $\mathrm{Ch}$ & CP5 & CFC5 & CP5 & $\mathrm{CCP} 2$ & CP5 & CCP5 & CP5 & $\mathrm{CCP} 2$ \\
\hline & TPR & 80.00 & 93.33 & 93.33 & 93.33 & 86.67 & 86.67 & 80.00 & 100 \\
\hline & FPR & 0 & 6.67 & 0 & 13.33 & 0 & 0 & 0 & 13.33 \\
\hline \multirow[b]{4}{*}{$\tilde{n}$} & Acc & 80.00 & 83.33 & 70.00 & 80.00 & 73.33 & 83.33 & 73.33 & 76.67 \\
\hline & $\mathrm{Ch}$ & $\mathrm{CZ}$ & $\mathrm{CP} 4$ & $\mathrm{CP} 1$ & CCP5 & CCP4 & $\mathrm{CFC4}$ & $\mathrm{CCP} 3$ & $\mathrm{CFCl}$ \\
\hline & TPR & 80.00 & 80.00 & 60.00 & 86.67 & 66.67 & 93.33 & 60.00 & 93.33 \\
\hline & FPR & 20.00 & 13.33 & 20.00 & 73.33 & 20.00 & 26.67 & 13.33 & 40.00 \\
\hline \multirow[b]{4}{*}{ 声 } & Acc & 90.00 & 80.00 & 80.00 & 76.67 & 80.00 & 80.00 & 76.67 & 86.67 \\
\hline & $\mathrm{Ch}$ & $\overline{F C 5}$ & CCP1 & $\mathrm{CFC} 3$ & $\mathrm{C} 4$ & FC5 & CFC5 & FC5 & $\mathrm{CP} 2$ \\
\hline & TPR & 93.33 & 93.33 & 93.33 & 93.33 & 60.00 & 66.67 & 60.00 & 73.33 \\
\hline & FPR & 13.33 & 33.33 & 33.33 & 40.00 & 0 & 0 & 6.67 & 0 \\
\hline \multirow[b]{4}{*}{$\tilde{n}$} & $\mathrm{Acc}$ & 83.33 & 93.33 & 76.67 & 90.00 & 80.00 & 96.67 & 76.67 & 96.67 \\
\hline & $\mathrm{Ch}$ & $\mathrm{CCP} 1$ & CCP1 & $\mathrm{C} 1$ & C3 & CFC1 & $\mathrm{C} 1$ & $\mathrm{CZ}$ & $\mathrm{CFC} 3$ \\
\hline & TPR & 73.33 & 86.67 & 60.00 & 80.00 & 73.33 & 93.33 & 66.67 & 100 \\
\hline & FPR & 6.67 & 0 & 6.67 & 0 & 13.33 & 0 & 13.33 & 6.67 \\
\hline
\end{tabular}

\begin{tabular}{|c|c|c|c|c|c|c|c|c|c|}
\hline & Acc & 76.67 & 90.00 & 73.33 & 93.33 & 70.00 & 83.33 & 73.33 & 96.67 \\
\hline \multirow[b]{3}{*}{ i } & $\mathrm{Ch}$ & $\mathrm{C} 3$ & CP3 & $\overline{\mathrm{C} 4}$ & CP1 & FC3 & CCP5 & CP1 & CP1 \\
\hline & TPR & 66.67 & 80.00 & 60.00 & 100 & 40.00 & 73.33 & 60.00 & 100 \\
\hline & $\begin{array}{l}\text { FPR } \\
\end{array}$ & 13.33 & 0 & 13.33 & 13.33 & 0 & 6.67 & 20.00 & 6.67 \\
\hline \multirow[b]{4}{*}{ is } & Acc & 73.33 & 93.33 & 76.67 & 96.67 & 73.33 & 90.00 & 76.67 & 93.33 \\
\hline & $\mathrm{Ch}$ & C3 & CFC5 & C5 & C4 & $\mathrm{FC1}$ & CCP5 & $\mathrm{C} 5$ & CFC5 \\
\hline & $\begin{array}{l}\text { TPR } \\
\end{array}$ & 66.67 & 86.67 & 66.67 & 93.33 & 60.00 & 86.67 & 60.00 & 93.33 \\
\hline & $\begin{array}{l}\text { FPR } \\
\end{array}$ & 20.00 & $\overline{0}$ & 13.33 & $\overline{0}$ & 13.33 & 6.67 & 6.67 & 6.67 \\
\hline \multirow[b]{4}{*}{$\infty$} & Acc & 70.00 & $\begin{array}{l}83.33 \\
\end{array}$ & 73.33 & 93.33 & 76.67 & 96.67 & 70.00 & 100 \\
\hline & $\mathrm{Ch}$ & FCZ & FC3 & CP5 & $\begin{array}{l}\mathrm{C} 5 \\
\end{array}$ & CP2 & CP2 & CP3 & FC3 \\
\hline & $\begin{array}{l}\text { TPR } \\
\end{array}$ & 80.00 & 73.33 & 80.00 & 86.67 & 93.33 & 100 & 80.00 & 100 \\
\hline & FPR & 40.00 & 6.67 & 33.33 & 0 & 40.00 & 6.67 & 40.00 & 0 \\
\hline \multirow[b]{4}{*}{ के } & Acc & 73.33 & 90.00 & 73.33 & 86.67 & 73.33 & 93.33 & 73.33 & 90.00 \\
\hline & $\begin{array}{ll}\mathrm{Ch} \\
\end{array}$ & $\mathrm{FC3}$ & $\overline{\mathrm{FC} 1}$ & CCP2 & CFC5 & CP3 & FCZ & CP3 & CP2 \\
\hline & $\begin{array}{l}\text { TPR } \\
\end{array}$ & 86.67 & 86.67 & 66.67 & 80.00 & 53.33 & 86.67 & 60.00 & 86.67 \\
\hline & $\begin{array}{l}\text { FPR } \\
\end{array}$ & 40.00 & 6.67 & 20.00 & $\begin{array}{l}6.67 \\
\end{array}$ & 6.67 & 0 & 13.33 & 6.67 \\
\hline \multirow{4}{*}{$\frac{0}{n}$} & Acc & 70.00 & 73.33 & 73.33 & 76.67 & 73.33 & 73.33 & 70.00 & 76.67 \\
\hline & $\mathrm{Ch}$ & FCZ & FC5 & CP5 & CFC2 & FCZ & FCZ & $\mathrm{CZ}$ & FCZ \\
\hline & $\begin{array}{l}\text { TPR } \\
\end{array}$ & 66.67 & 66.67 & 66.67 & 80.00 & 80.00 & 73.33 & 66.67 & 66.67 \\
\hline & FPR & 26.67 & 20.00 & 20.00 & 26.67 & 33.33 & 26.67 & 26.67 & 13.33 \\
\hline \multirow{4}{*}{$\bar{n}$} & Acc & 70.00 & 80.00 & 73.33 & 83.33 & 73.33 & 80.00 & 90.00 & 93.33 \\
\hline & $\mathrm{Ch}$ & CCP3 & FC5 & CCP5 & $\mathrm{CP} 2$ & FCZ & FC5 & FC5 & C5 \\
\hline & $\begin{array}{l}\text { TPR } \\
\end{array}$ & 73.33 & 66.67 & 80.00 & 73.33 & 86.67 & 73.33 & 100 & 100 \\
\hline & FPR & 33.33 & 6.67 & 33.33 & 6.67 & 40.00 & 13.33 & 20.00 & 13.33 \\
\hline
\end{tabular}

From Table 3, the classification results of four directions for detecting intention of movement lie within the range of $70.00 \%$ to $100.00 \%$. Maximum classification accuracy for these four directions are obtained from different electrodes, namely CFC5 and CCP1 with classification accuracy of $93.33 \%$ (direction toward 3), CP5 and C4 with classification accuracy of $96.67 \%$ (direction toward 6), $\mathrm{C} 1$ and $\mathrm{CP} 2$ with classification accuracy of $96.67 \%$ (direction toward 9) and FC3 with classification accuracy of $100.00 \%$ (direction toward 12). In all four directions, features extracted using spatial filter LAP contributed for the maximum classification results.

TABLE 4: Classification results of detection intention of movement for direction toward 3, 6, 9 and 12 using QDA classifier on average of Power Spectrum in alpha $(\alpha)$, beta $(\beta)$ and gamma $(\gamma)$ band feature.

\begin{tabular}{|c|c|c|c|c|c|c|c|c|c|}
\hline \multirow{3}{*}{ 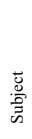 } & \multirow{3}{*}{$\begin{array}{l}\text { 总 } \\
\text { हूँ } \\
\text { हूँ }\end{array}$} & \multicolumn{8}{|c|}{$\begin{array}{l}\text { Maximum Classification Accuracy, True Positive Rate (TPR) and False Positive Rate (FPR) } \\
\text { for direction } 3,6,9 \text { and } 12(\%)\end{array}$} \\
\hline & & \multicolumn{2}{|c|}{ Direction 3} & \multicolumn{2}{|c|}{ Direction 6} & \multicolumn{2}{|c|}{ Direction 9} & \multicolumn{2}{|c|}{ Direction 12} \\
\hline & & CAR & LAP & CAR & LAP & CAR & LAP & CAR & LAP \\
\hline \multirow[b]{4}{*}{$\bar{\omega}$} & Acc & 70.00 & 76.67 & \begin{tabular}{|c|}
76.67 \\
\end{tabular} & 73.33 & 86.67 & 83.33 & 73.33 & \begin{tabular}{|c|}
76.67 \\
\end{tabular} \\
\hline & $\mathrm{Ch}$ & CCP4 & $\mathrm{FC} 2$ & CP5 & CFC4 & $\mathrm{FC} 4$ & CFC4 & $\mathrm{C} 4$ & CCP1 \\
\hline & TPR & 66.67 & 66.67 & 80.00 & 66.67 & 86.67 & 80.00 & 93.33 & 53.33 \\
\hline & FPR & 26.67 & 13.33 & 26.67 & 20.00 & 13.33 & 13.33 & 46.67 & 0 \\
\hline \multirow[b]{4}{*}{ i } & Acc & 80.00 & 96.67 & 90.00 & 93.33 & 96.67 & 93.33 & 90.00 & 90.00 \\
\hline & $\mathrm{Ch}$ & CPZ & CCP3 & CP5 & CCP2 & CP5 & CCP2 & CP5 & CCP5 \\
\hline & TPR & 100 & 100 & 93.33 & 86.67 & 93.33 & 86.67 & 80.00 & 80.00 \\
\hline & FPR & 40.00 & 6.67 & 13.33 & 0 & 0 & 0 & 0 & 0 \\
\hline \multirow[b]{4}{*}{$\tilde{n}$} & Acc & 73.33 & 70.00 & 66.67 & 70.00 & 60.00 & 73.33 & 66.67 & 70.00 \\
\hline & $\mathrm{Ch}$ & $\mathrm{C} 2$ & CCP3 & $\mathrm{FCl}$ & CCP5 & CCP2 & $\mathrm{CZ}$ & C4 & CCP5 \\
\hline & TPR & 66.67 & 66.67 & 60.00 & 73.33 & 40.00 & 86.67 & 40.00 & 66.67 \\
\hline & FPR & 20.00 & 26.67 & 26.67 & 33.33 & 20.00 & 40.00 & 6.67 & 26.67 \\
\hline \multirow[b]{4}{*}{ 声 } & Acc & 76.67 & 80.00 & 73.33 & 73.33 & \begin{tabular}{|l|l|}
83.33 \\
\end{tabular} & 80.00 & 86.67 & 90.00 \\
\hline & $\mathrm{Ch}$ & FC5 & CFC5 & FC3 & $\mathrm{C} 3$ & $\mathrm{CP1}$ & CFC5 & C1 & $\mathrm{FC} 2$ \\
\hline & TPR & 80.00 & 86.67 & 73.33 & 86.67 & 73.33 & 73.33 & 80.00 & 86.67 \\
\hline & FPR & 26.67 & 26.67 & 26.67 & 40.00 & 6.67 & 13.33 & 6.67 & 6.67 \\
\hline \multirow[b]{4}{*}{ 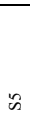 } & Acc & 86.67 & 90.00 & \begin{tabular}{|c|}
76.67 \\
\end{tabular} & 96.67 & 83.33 & 93.33 & $\begin{array}{l}76.67 \\
\end{array}$ & 86.67 \\
\hline & $\mathrm{Ch}$ & FC3 & CCP1 & $\mathrm{CP} 1$ & CFC1 & $\mathrm{C} 1$ & CCP3 & $\mathrm{CZ}$ & CFC3 \\
\hline & TPR & 86.67 & 93.33 & 73.33 & 93.33 & $\begin{array}{l}73.33 \\
\end{array}$ & 86.67 & 73.33 & 93.33 \\
\hline & FPR & 13.33 & 13.33 & 20.00 & 0 & 6.67 & 0 & 20.00 & 20.00 \\
\hline \multirow[b]{4}{*}{ ஜ } & Acc & 70.00 & 90.00 & 70.00 & 96.67 & 73.33 & 83.33 & 66.67 & 96.67 \\
\hline & $\mathrm{Ch}$ & CCP4 & CP4 & C4 & FC4 & $\mathrm{FC} 3$ & CFC5 & CFC3 & C5 \\
\hline & TPR & 53.33 & 100 & 73.33 & 93.33 & 66.67 & 86.67 & 73.33 & 93.33 \\
\hline & FPR & 13.33 & 20.00 & 33.33 & 0 & 20.00 & 20.00 & 40.00 & 0 \\
\hline
\end{tabular}




\begin{tabular}{|c|c|c|c|c|c|c|c|c|c|}
\hline \multirow[b]{4}{*}{ in } & Acc & 80.00 & 96.67 & 73.33 & 96.67 & 73.33 & 93.33 & 73.33 & 96.67 \\
\hline & $\mathrm{Ch}$ & CP5 & CCP5 & C3 & CCP5 & CCP4 & CFC5 & $\mathrm{CCP} 3$ & CCP5 \\
\hline & TPR & 86.67 & 100 & 60.00 & 100 & 66.67 & 86.67 & 66.67 & 100 \\
\hline & FPR & 26.67 & 6.67 & 13.33 & 6.67 & 20.00 & 0 & 20.00 & 6.67 \\
\hline \multirow[b]{4}{*}{$\stackrel{\infty}{\infty}$} & Acc & 73.33 & 70.00 & 70.00 & 93.33 & 73.33 & 93.33 & 73.33 & 93.33 \\
\hline & $\mathrm{Ch}$ & СCP2 & CP2 & FCZ & CP1 & CP4 & $\mathrm{FC} 2$ & CP1 & CP3 \\
\hline & $\begin{array}{l}\text { TPR } \\
\end{array}$ & 80.00 & 86.67 & 66.67 & 93.33 & 86.67 & 93.33 & 73.33 & 86.67 \\
\hline & FPR & 33.33 & 46.67 & 26.67 & 6.67 & 40.00 & 6.67 & 26.67 & 0 \\
\hline \multirow[b]{4}{*}{ in } & Acc & 73.33 & 86.67 & 73.33 & 86.67 & 63.33 & 90.00 & 70.00 & 80.00 \\
\hline & $\mathrm{Ch}$ & CFCl & CP1 & $\mathrm{CCP} 1$ & CP2 & $\overline{\mathrm{C} 3}$ & FCZ & $\mathrm{C} 3$ & CP1 \\
\hline & TPR & 53.33 & 100 & 73.33 & 80.00 & 73.33 & 80.00 & 60.00 & 80.00 \\
\hline & FPR & 6.67 & 26.67 & 26.67 & 6.67 & 46.67 & 0 & 13.33 & 20.00 \\
\hline \multirow{4}{*}{$\frac{0}{n}$} & Acc & 70.00 & 70.00 & 70.00 & 70.00 & 70.00 & 70.00 & 66.67 & 73.33 \\
\hline & $\mathrm{Ch}$ & $\mathrm{CFC2}$ & $\begin{array}{l}\mathrm{CP} 4 \\
\end{array}$ & $\mathrm{FC1}$ & CCP1 & $\mathrm{C} 4$ & $\overline{\mathrm{CCP} 4}$ & $\mathrm{CZ}$ & $\mathrm{FC} 2$ \\
\hline & TPR & 60.00 & 60.00 & 60.00 & 53.33 & 66.67 & 73.33 & 80.00 & 60.00 \\
\hline & FPR & 20.00 & 20.00 & 20.00 & 13.33 & 26.67 & 33.33 & 46.67 & 13.33 \\
\hline \multirow[b]{4}{*}{$\bar{n}$} & Acc & 70.00 & 80.00 & 70.00 & 76.67 & 63.33 & 66.67 & 93.33 & 96.67 \\
\hline & $\mathrm{Ch}$ & CCP5 & FC3 & CFC2 & $\mathrm{FC} 2$ & $\mathrm{C} 2$ & C5 & FC5 & CFC5 \\
\hline & $\begin{array}{l}\text { TPR } \\
\end{array}$ & 66.67 & 73.33 & 66.67 & 60.00 & 66.67 & 66.67 & 93.33 & 93.33 \\
\hline & $\begin{array}{l}\text { FPR } \\
\end{array}$ & 26.67 & 13.33 & 26.67 & 6.67 & 40.00 & 33.33 & 6.67 & 0 \\
\hline
\end{tabular}

Table 4 indicates the classification results of four directions for detecting intention of movement lie within the range of $60.00 \%$ to $96.67 \%$. Maximum classification accuracy for these four directions are obtained from different electrodes, namely CCP3 and CCP5 with classification accuracy of $96.67 \%$ (direction toward 3), CFC1, CCP5 and FC4 with classification accuracy of 96.67\% (direction toward 6), CCP3,CFC5 and FC2 with classification accuracy of $93.33 \%$ (direction toward 9) and CCP5 and C5 with classification accuracy of $96.67 \%$ (direction toward 12). In all four directions, features extracted using spatial filter LAP contributed for the maximum classification results.

\section{Conclusion}

In this paper we have demonstrated the feasibility of detecting motor imagery and intention of movement using right wrist movement in multiple directions namely, direction towards $3,6,9$ and 12 . This is significantly supported by ERSP results that evidently detected ERD and ERS in both intention and imagination of movement in all directions.

The classification results from this paper also highlights the comparison of using two type of spatial filters namely CAR and LAP, wherein the LAP filter outperforms CAR. This finding is supported by $\mathrm{Ng}$ and Raveendran (2007). In their study which based on motor imagery paradigm, LAP outperformed other spatial filter using ERD/ERS from different hemispheres. On the other hand our findings contradict the finding by Alhaddad (2012). In his work (based on P300 paradigm) CAR outperformed LAP. Nonetheless, both CAR and LAP referencing are superior to the ear reference [10]. Apart from that the classification results, the importance of implementation of high density electrodes is also highlighted.

\section{References}

1 J. R. Wolpaw, N. Birbaumer, D. J. McFarland, G. Pfurtscheller, and T. M. Vaughan, "Braincomputer interfaces for communication and control," Clin. Neurophysiol., vol. 113, no. 6, pp. 767-791, Jun. 2002.
2 J. D. R. Millán, R. Rupp, G. R. Müller-Putz, R. Murray-Smith, C. Giugliemma, M. Tangermann, C. Vidaurre, F. Cincotti, a Kübler, R. Leeb, C. Neuper, K.-R. Müller, and D. Mattia, "Combining Brain-Computer Interfaces and Assistive Technologies: State-of-the-Art and Challenges," Front. Neurosci., vol. 4, no. September, pp. 1-15, Jan. 2010.

3 K. K. Ang, J. Yu, and C. Guan, "Extracting effective features from high density nirs-based BCI for assessing numerical cognition,” 2012 IEEE Int. Conf. Acoust. Speech Signal Process, pp. 22332236, Mar. 2012.

4 M. J. Alhaddad, "Common Average Reference (CAR) Improves P300 Speller,'International Journal of Engineering and Technology, vol. 2, no. 3, 2012.

5 X. Lei, P. Yang, P. Xu, T. Liu, and D. Yao, "Common Spatial Pattern Ensemble Classifier and Its Application in Brain-Computer Interface," Journal of Electronic Science and Technology of China, vol. 7, no. 1, pp. 17-21, 2009.

6 C. Sannelli, C. Vidaurre, K. Müller, and B. Blankertz, "Common Spatial Pattern Patches - an Optimized Filter Ensemble for Adaptive BCIs," International Journal of Bioelectromagnetism, vol. 13, no. 3, pp. 161-162, 2011.

7 T. Solis-Escalante, G. Müller-Putz, and G. Pfurtscheller, "Overt foot movement detection in one single Laplacian EEG derivation," J. Neurosci. Methods, vol. 175, no. 1, pp. 148-53, Oct. 2008.

8 J. Lu, D. J. McFarland, and J. R. Wolpaw, "Adaptive Laplacian filtering for sensorimotor rhythm-based brain-computer interfaces.," J. Neural Eng., vol. 10, no. 1, p. 016002, Feb. 2013.

9 Teplan, M, "Fundamentals of EEG Measurement," Measurement Science Review, 2(2),1-11,2002.

10 D. J. McFarland, L. M. McCane, S. V. David, and J. R. Wolpaw, "Spatial filter selection for EEGbased communication," Electroencephalogr. Clin. Neurophysiol., vol. 103, no. 3, pp. 386-394, Sep. 1997.

11 G. Pfurtscheller and F. H. Lopes da Silva "Eventrelated EEG/MEG synchronization and desynchronization: basic principles," Clin. Neurophysiol., vol. 110, no. 11, pp. 1842-57,1999.

12 Delorme, A. \&Makeig, S“EEGlab: An Open Source Toolbox For Analysis Of Single-Trial EEG Dynamics Including Independent Component Analysis," Journal Of Neuroscience Methods, 134,9-21,2004.

13 M. Ferdjallah and R. E. Barr. "On the Unit Circle for the Removal of Power line Noise from Biomedical Signals," vol. 41, no. 6, pp. 529-536, 1994.

14 V. B. Deepa\& P. Thangaraj. "A study on classification of EEG Data using the Filters," vol. 2, no. 4, pp. 94-96, 2011

15 H. Lakany and B. a Conway, "Classification of Wrist Movements using EEG-based Wavelets Features.," Conf. Proc. IEEE Eng. Med. Biol. Soc., vol. 5, pp. 5404-5407, 2005. 
16 Lakany, H. and Conway, B.A. "Comparing EEG patterns of actual and imaginary wrist movements A machine learning approach," Proceedings of the first ICGST International Conference on Artificial Intelligence and Machine Learning AIML 05, 5 . ICGST, Cairo, Egypt, pp. 124-127, 2005.

17 I. Dokare and N. Kant, "Performance Analysis of SVM, k-NN and BPNN Classifiers for Motor Imagery," vol. 10, no. 1, pp. 19-23, 2014.

18 C. Liu, H. Wang, and Z. Lu, "EEG Classification for Multiclass Motor Imagery BCI," Chinese Control and Decision Conference, pp. 4450-4453, 2013.

19 S. Bhattacharyya, A. Khasnobish, S. Chatterjee, A. Konar, and D. N. Tibarewala, "Performance analysis of LDA, QDA and KNN algorithms in left-right limb movement classification from EEG data," Int. Conf. Syst. Med. Biol. ICSMB 2010 Proc., no. December, pp. 126-131, 2010.

20 O. Carrera-león, J. M. Ramirez, V. Alarcon-aquino, M. Baker, D. D. Croz-baron, and P. Gomez-gil, "A Motor Imagery BCI Experiment using Wavelet Analysis and Spatial Patterns Feature Extraction," pp. 18-20.

21 C. Elkan, "Evaluating Classifiers", University of San Diego, California, retrieved [01-05-2013] from http://cseweb.ucsd.edu/ elkan/250B/classifiereval. pdf

22 R. Palaniappan, "Biological Signal Analysis," Ventus Publishing, Denmark, 2010.

23 H. Hwang, K. Kwon, and C. Im, "Neurofeedbackbased motor imagery training for brain - computer interface (BCI )," vol. 179, pp. 150-156, 2009.

24 S. C. $\mathrm{Ng}$ and P. Raveendran, "Comparison of different Montages on to $\{E E G\}$ classification," 3rd Kuala Lumpur Int. Conf. Biomed. Eng. 2006, vol. 15, pp. 365-368, 2007. 